\title{
Elucidating the Dynamics of Polymer Transport through Nanopores using Asymmetric Salt
}

\section{Concentrations}

Martin Charron, $\neq$ Lucas Philipp, $\neq$ Liqun He, Vincent Tabard-Cossa*

Department of Physics, University of Ottawa, Ontario, Canada

Keywords: Nanopore, DNA Translocation, Salt Gradient, Tension Propagation, Polymer

Transport

Abstract: While notable progress has been made in recent years both experimentally and theoretically in understanding the highly complex dynamics of polymer capture and transport through nanopores, there remains significant disagreement between experimental observation and theoretical prediction that needs to be resolved. Asymmetric salt concentrations, where the concentrations of ions on each side of the membrane are different, can be used to enhance capture rates and prolong translocation times of polymers translocating through a nanopore from the low salt concentration reservoir, which are both attractive features for single-molecule analysis. However, since asymmetric salt concentrations affect the electrophoretic pull inside and outside the pore differently, it also offers a useful control parameter to elucidate the otherwise inseparable physics of the capture and translocation process. In this work, we attempt to paint a complete 
picture of the dynamics of polymer capture and translocation in both symmetric and asymmetric salt concentration conditions by reporting the dependence of multiple translocation metrics on voltage, polymer length, and salt concentration gradient. Using asymmetric salt concentration conditions, we experimentally observe the predictions of tension propagation theory and verify the significant impact of the electric field gradient on pre-stretching polymers on approach to the pore.

Elucidating the physics that govern nanopore transport phenomena is a fertile field of research and is important for increasing our ability to design strategies for controlling molecular passage from which numerous technological applications can be built. On top of the spectacular innovations in DNA sequencing technology, ${ }^{1-5}$ nanopores promise radical advances in the life sciences and medicine with ultra-sensitive, point-of-care diagnosis of disease,${ }^{6-12}$ identification and sequencing of full-length proteins, ${ }^{13-16}$ and in next-generation information storage with decoding of digital data from sequence-controlled polymers. ${ }^{17,18}$ To date however, while multiple theories have been developed to describe both the capture ${ }^{19,20}$ and translocation ${ }^{21-26}$ processes, only a few experimental studies ${ }^{27-33}$ have attempted to validate the proposed concepts, with varying degrees of agreement. This is in large part due to the complex nature of the translocation dynamics, which occur as a multi-step, highly non-equilibrium process dictated mainly by forces over which experimental control has been challenging or impossible, making verification of theoretical ideas particularly difficult. Only just recently has the first clear experimental confirmation of tension-propagation theory been provided, obtained through the measurement of the two-step, non-constant and non-monotonic velocity profile of nanostructured DNA molecules undergoing translocation. ${ }^{34}$ Further experimental work is however needed to provide a more 
complete picture of the dynamics of polymer transport and to deliver better insights on how to design or improve many sensing and sequencing schemes.

Asymmetric salt concentration (ASC) conditions, in which a nanopore separates two reservoirs of different salt concentrations, offer a flexible method to probe the dynamics of transport through nanopores. A non-uniform distribution of salt concentration across a nanopore system results in a correspondingly non-uniform modulation of both the electrophoretic mobility and the electric field strength, which in turn affect the dynamics of capture and translocation of charged molecules through nanopores. ${ }^{33,35-41}$

Using a simplified three resistors in series model to predict the electrical response of the nanopore and its two access regions, the cis- and trans- sides of Figure 1a, gives the appropriate level of insight into the system's electrical characteristics in different ASC conditions. As per convention a molecule traverses the pore from the cis- to the trans-side of the membrane. In ASC conditions, the cis-side voltage drop $\Delta V_{c i s}$ and electric field $E_{c i s}$ grow monotonically with salt concentration ratio $\mathrm{C}_{\text {trans }} / \mathrm{C}_{c i s}$, whereas the opposite is true for the trans-side. Molecules in the lowsalt reservoir are therefore subject to a higher field than in symmetric salt concentrations (SSC) conditions, and similarly molecules in high-salt are subject to a lower field. Conversely, the voltage drop inside the pore $\Delta V_{\text {pore }}$ is expected to be largest in SSC conditions and reduced under any ASC condition. See Section S1 of the Supporting Information for more details and derivations.

Interestingly, unlike the effect of modulating voltage or pore diameter, ASC conditions provide a simple method by which to decouple capture rate and translocation velocity; Measurements with molecules in lower salt concentration than the trans side $\left(\mathrm{C}_{\text {trans }} / \mathrm{C}_{\text {cis }}>1\right)$ have demonstrated both increased capture rate and decreased translocation velocity, ${ }^{33,35-41}$ whereas 
these quantities are positively correlated when modulated by other standard means (e.g. tuning the voltage, salt concentration, ${ }^{42}$ pore $\operatorname{size}^{32}$ or temperature $\left.{ }^{43}\right)$. The increased capture rate when $\mathrm{C}_{\text {trans }} / \mathrm{C}_{\text {cis }}>1$ is understood to be a direct consequence of higher $E_{c i s},{ }^{33,44}$ whereas the origin of the slower translocation remains unclear and has not been carefully tested experimentally. Factors believed to decrease the translocation velocity include lower $\Delta V_{\text {pore }},{ }^{33}$ and cation accumulation inside the pore retarding motion through increased electro-osmotic flow opposing the motion of the negatively charged analyte. ${ }^{33,36}$

In this work, we use ASC conditions as a sensitive method to independently modulate the different forces impacting the capture and translocation of double-stranded DNA fragments through solid-state nanopores in the 5 to $10 \mathrm{~nm}$ pore size range, made in $\sim 10 \mathrm{~nm}$ thick silicon nitride membranes. We report the dependence on applied voltage, DNA length, and salt concentration ratio of multiple metrics including capture rate, mean and standard deviation of translocation times, and the fraction of folded translocations (results of the latter are only shown in section S8 of the Supporting Information). In doing so, we reveal that the underlying physics governing the capture and translocation processes under ASC conditions are fundamentally the same as in SSC, though the magnitude and balance of forces inside and outside the pore differ due to changes in the electric field profile and electrophoretic mobility throughout the system.

Briefly, we find that capture in ASC conditions is described by the same two regimes found in SSC conditions, namely the diffusion- and barrier-limited regimes, ${ }^{20}$ but that ASC conditions modulate the voltage and polymer length at which capture transitions from one regime to the other. We then validate that the translocation times scale with voltage and polymer-length as postulated by iso-flux tension propagation formalisms, ${ }^{24}$ but reveal different scaling exponents between ASC and SSC conditions. Using higher-order translocation time statistics, we further demonstrate that 
ASC conditions alter polymer conformations and elongations prior to translocation, which helps explain the different translocation time scalings observed in ASC and SSC conditions. These results highlight the dual effect of the non-uniform external field in first elongating polymers before their arrival at the pore entrance and then compressing them against the nanopore membrane prior to the start of their translocation. ${ }^{45-47}$ The depth of the data presented provides experimental confirmation of previously proposed theoretical concepts, and delivers new insights to design ways to control the motion and capture of target molecules.

\section{Results and Discussion}

\section{Nanopore Capture and Translocation}

The capture and translocation of polymers into nanopores is a complex nonequilibrium process resulting from coupled electrohydrodynamic and diffusion processes which occur on a wide variety of timescales spanning both sides of the polymer relaxation time. The electric field inside and outside the pore drives most of the directed transport mechanisms. ${ }^{19,20}$ The magnitude of the electric field is largest inside the nanopore due to its nanoscopic geometry and high electrical resistance, whereas the field outside the nanopore decays away from the pore approximately as $\sim 1 / \mathrm{r}^{2}{ }^{20,48,49}$ Naturally, the local details of the electric field strength and profile depend strongly on nanopore geometry and the distribution of ions in the system.

The complete capture and translocation process of a polymer can be described using the

five following steps: i) The polymer diffuses from the solution bulk close to the nanopore capture volume, a hemisphere-like volume inside which the field-induced drift is stronger than thermal motion, ${ }^{33,48,49}$ ii) The polymer electrophoretically drifts towards the pore, during which it is 
elongated by the electric field gradient, ${ }^{45-47}$ until it arrives at the pore; iii) The polymer at the pore entrance is compressed against the nanopore membrane by the electric field until threading begins either by an end or a folded segment of the polymer; ${ }^{46,47}$ iv) Due to the high electric field inside the pore, the polymer undergoes non-equilibrium deformation during translocation. As consecutive segments are pulled inside the pore, the fraction of the polymer still on the cis-side is being deformed with a growing mobile segment as the tension propagates along its contour length; $22-24,34,50,51$ v) Once the tension reaches the polymer end, the polymer continues translocation but with a shrinking $c i s$-side segment fully under tension until the polymer is fully threaded..$^{22-24,34,50,51}$

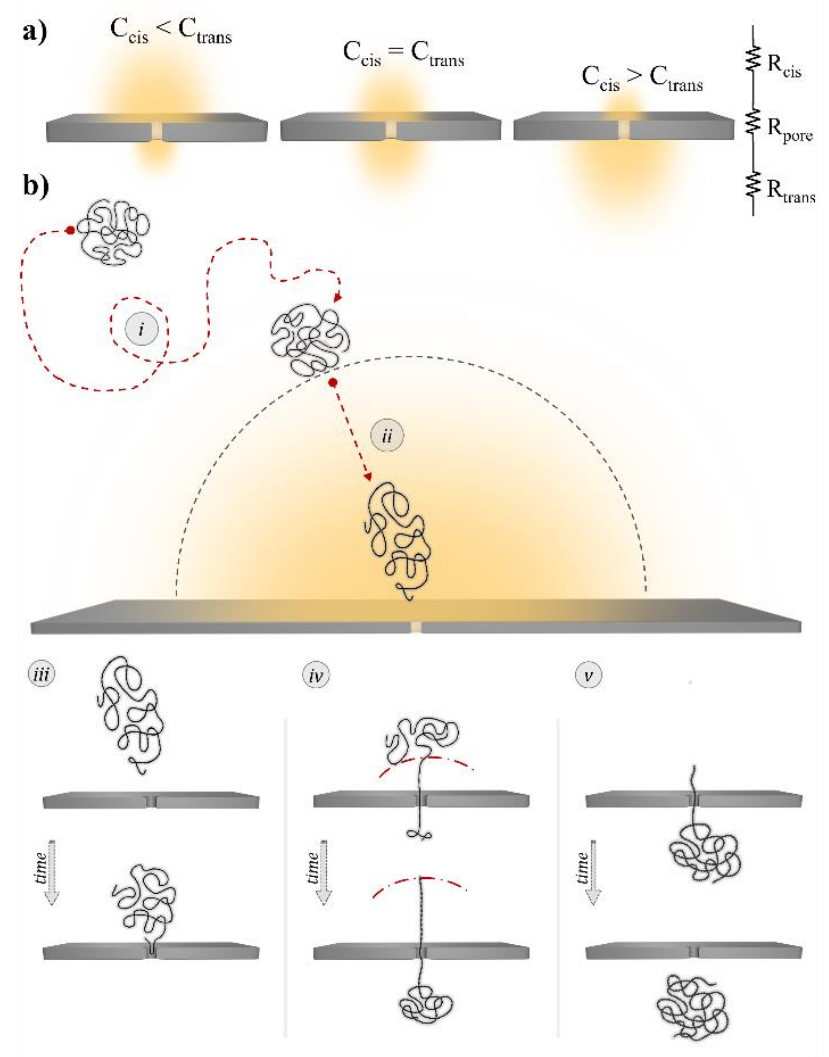

Figure 1. (a) Schematic description of the effect of asymmetric salt conditions on the resulting electric field. (b) Schematic description of capture and translocation steps: (i) Molecules diffuse close to the pore; (ii) Molecules drift to the pore mouth due to larger electric field; (iii) A polymer segment finds and enters the pore, thereby initiating 
translocation; (iv) Tension propagation: A growing fraction of the polymer is under tension/motion. The tension front is represented by a red dashed line; (v) Post-propagation: All monomers on cis-side move towards the pore;

Note that only the strongly driven translocation regime has been described above, and not the weakly driven regime, ${ }^{52}$ in which the translocation steps $i v$ and $v$ occur on timescales comparable to or longer than the relaxation time of the polymer. Since the majority of experimental work with solid-state nanopores occurs in the strongly driven regime, we focus our efforts there.

The five capture and translocation steps are illustrated in Figure $1 \mathrm{~b}$ for clarity. Note that the expected polymer conformations throughout the transport process are determined by the relative timescales of each of these steps, which depend strongly on the forces at play: The magnitude of the external field $E_{c i s}$ impacts the duration of step $i i$, and therefore helps in determining how elongated a polymer is expected to be when arriving at the pore. Similarly, the magnitude of the field inside the pore impacts the duration of step iii, i.e. how quickly a molecule can surpass the free-energy barrier at the pore mouth, and therefore helps in determining how much a polymer will compress against the nanopore membrane before beginning translocation.

\section{Capture Scaling}

In order to investigate how the capture process is affected by different electric field profiles resulting from ASC conditions, we first describe the scaling of capture rate with applied voltage $(\Delta V)$ and polymer length $(N)$ in both ASC and SSC conditions. As described elsewhere, capture rate in standard SSC conditions is well described by both the barrier-limited regime for short polymers or low voltages, and by the diffusion-limited regime for long polymers or high voltages 
(also termed drift-limited regime ${ }^{19,28}$ ), in which the rate-limiting step is the polymer overcoming a largely entropic, free-energy barrier and diffusing into the capture volume, respectively. ${ }^{19,20,48,53}$

$$
R \equiv \frac{J}{c} \propto\left\{\begin{aligned}
N^{\gamma} e^{\delta \Delta V}, & N, \Delta V<N^{*}, \Delta V^{*} \\
\mu_{e} \Delta V, & N, \Delta V>N^{*}, \Delta V^{*}
\end{aligned}\right.
$$

Here, $R$ is the normalized capture rate, defined as the ratio of capture rate $J$ and polymer concentration $c, \mu_{e}$ is the electrophoretic mobility of the charged polymer, and $\gamma$ and $\delta$ are scaling coefficients. The regime-transition polymer length and voltage values are denoted by $N^{*}$ and $\Delta V^{*}$, are interdependent, and both depend strongly on experimental parameters. ${ }^{19,20}$ Note that since we use double-stranded DNA (dsDNA) as our model linear polymer, and its electrophoretic mobility is length independent above a few hundred bases,${ }^{54}$ its capture rate is expected to scale as $R \sim N^{0} \Delta V^{1}$ in the diffusion-limited regime. ${ }^{28,29,33}$

To test the validity of the voltage scaling of Equation 1 for polymer capture in ASC conditions, we performed experiments using $0.25 \mathrm{nM}$ of $10 \mathrm{kbp}$ dsDNA in a $5.3 \mathrm{~nm}$ diameter nanopore, under applied voltages ranging from $50 \mathrm{mV}$ to $600 \mathrm{mV}$, in three different $\mathrm{LiCl}$ salt conditions: $\mathrm{SSC}\left(\mathrm{C}_{\text {trans }} / \mathrm{C}_{c i s}=0.45 \mathrm{M} / 0.45 \mathrm{M}=1\right)$; capture-promoting $\mathrm{ASC}\left(\mathrm{C}_{\text {trans }} / \mathrm{C}_{\text {cis }}=3.6 \mathrm{M} / 0.45\right.$ $\mathrm{M}>1)$; and capture-opposing $\mathrm{ASC}\left(\mathrm{C}_{\text {trans }} / \mathrm{C}_{\text {cis }}=0.45 \mathrm{M} / 3.2 \mathrm{M}<1\right)$. Figures 2a-c show representative current traces acquired under a bias of $400 \mathrm{mV}$ in SSC, capture-promoting and capture-opposing ASC conditions, respectively. At each voltage, the inter-event time distribution was extracted, and the corresponding capture rate was determined, ${ }^{29}$ as shown in Figure 2d. 

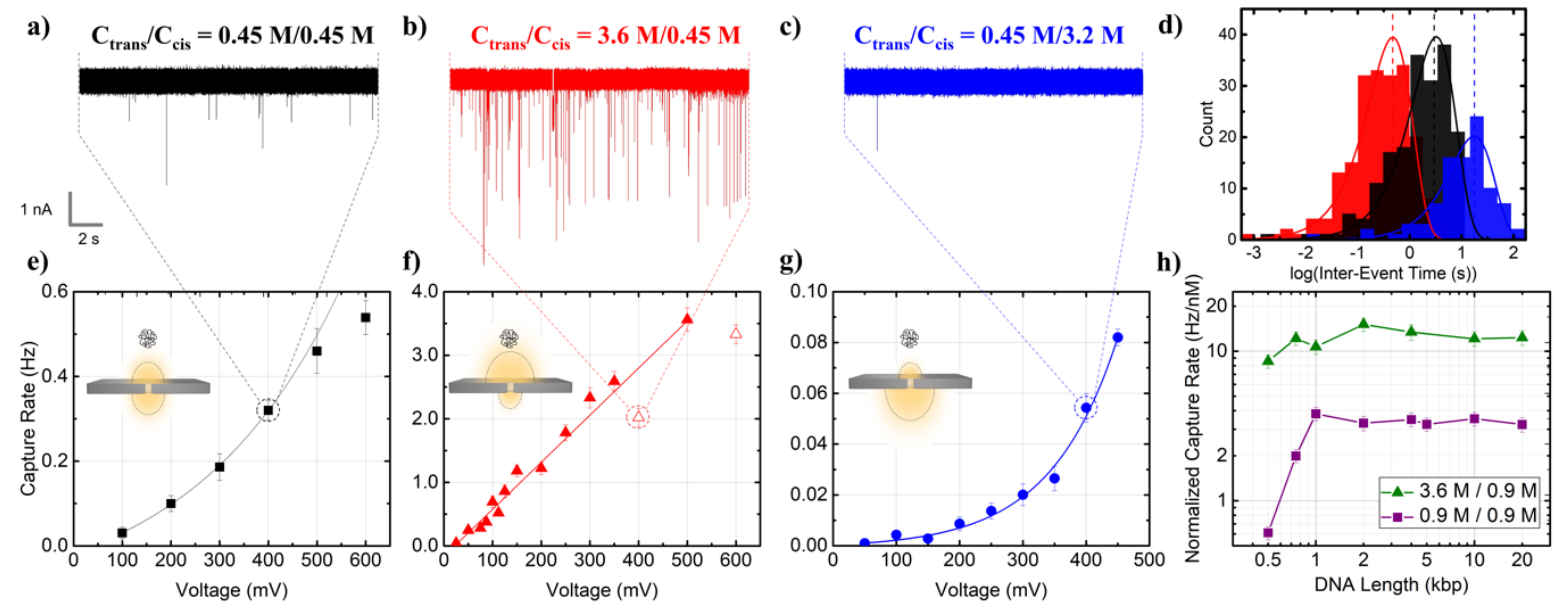

Figure 2. Comparison of capture kinetics in symmetric and asymmetric salt concentration conditions.

Representative ionic current traces for $0.25 \mathrm{nM}$ of $10 \mathrm{kbp}$ dsDNA under $400 \mathrm{mV}$ in a $5.3 \mathrm{~nm}$ pore in a $10 \mathrm{~nm}$ thick $\mathrm{SiN}$ membrane under (a) symmetric salt conditions $(0.45 \mathrm{M} \mathrm{LiCl})$; (b) capture-promoting salt conditions $\left(\mathrm{C}_{\text {trans }} / \mathrm{C}_{\text {cis }}\right.$ $>1)$; (c) and capture-opposing salt conditions $\left(\mathrm{C}_{\text {trans }} / \mathrm{C}_{c i s}<1\right)$. Data sampled at $4.17 \mathrm{MHz}$, analyzed, and displayed at $300 \mathrm{kHz}$ with a low-pass Bessel filter. (d) Inter-event time distribution fitting for the data at $400 \mathrm{mV}$ shown in a), b) and c) with corresponding colors, where capture rate is extracted by fitting the log-transform of a Poisson distribution. (e) Voltage dependence of capture rate in SSC conditions ( $0.45 \mathrm{M} \mathrm{LiCl}$, black squares); (f) capturepromoting ASC conditions $\left(\mathrm{C}_{\text {trans }} / \mathrm{C}_{c i s}>1\right.$, red triangles); (g) and capture-opposing ASC conditions $\left(\mathrm{C}_{\text {trans }} / \mathrm{C}_{c i s}<1\right.$, blue circles). (h) Normalized capture rate versus DNA length in an $8.5 \mathrm{~nm}$ pore in symmetric $0.9 \mathrm{M} \mathrm{LiCl}$ salt conditions (purple squares) and 4x capture-promoting ASC conditions (green triangles) under a $400 \mathrm{mV}$ bias. Error bars result from assuming Poisson statistics for capture rate uncertainty and a conservative $10 \%$ DNA concentration uncertainty. Note that some intra-pore capture rate variations are present, which we attribute to uncontrolled variability sources. ${ }^{29}$ Data deemed as outliers are displayed as hollow, as shown in f), and ignored during fitting.

As a control, Figure 2e shows the voltage dependence of the capture rate of $10 \mathrm{kbp}$ dsDNA in symmetric $0.45 \mathrm{M} \mathrm{LiCl}$ conditions for this $5.3 \mathrm{~nm}$ nanopore. A subtle nonlinear trend can be observed for $\Delta V \leq 400 \mathrm{mV}$, which fits well to an exponential function. For $\Delta V>400 \mathrm{mV}$, the data does not fit well to an exponential fit and is expected instead to be described by a linear voltage 
dependence, as supported and discussed in the following section. These trends are expected from Equation 1 in SSC conditions, ${ }^{28,29,33,48}$ with the change of capture regime from barrier-limited to diffusion-limited occurring around $\Delta V^{*} \approx 400 \mathrm{mV}$.

Figure $2 \mathrm{f}$ shows the voltage dependence of the capture rate in the $\mathrm{C}_{\text {trans }} / \mathrm{C}_{c i s}=3.6 \mathrm{M} / 0.45$ $\mathrm{M}>1$ ASC condition on the same pore. As expected, the measured capture rates are appreciably higher than in SSC conditions (e.g. $3.56 \mathrm{~Hz}$ vs. $0.46 \mathrm{~Hz}$ at $500 \mathrm{mV}$ ). The capture rate in this capture-promoting case exhibits a linear voltage dependence for the entire voltage range, characteristic of the diffusion-limited regime, even at very low voltages $\left(\Delta V^{*}<50 \mathrm{mV}\right)$. Conversely, Figure $2 \mathrm{~g}$ shows that when the polymer is located on the high salt concentration side of a comparable salt gradient $\left(\mathrm{C}_{\text {trans }} / \mathrm{C}_{c i s}=0.45 \mathrm{M} / 3.2 \mathrm{M}<1\right)$, the capture rate is drastically reduced and a clear exponential voltage dependence is observed throughout the entire voltage range investigated, characteristic of the barrier-limited regime $\left(\Delta V^{*}>500 \mathrm{mV}\right)$.

Next, we explored the scaling of capture with polymer length, by measuring the capture rates of dsDNA ranging from 500 to $20,000 \mathrm{bp}$ in an $8.5 \mathrm{~nm}$ pore under a fixed $400 \mathrm{mV}$ bias in two different $\mathrm{LiCl}$ salt conditions. Figure $2 \mathrm{~h}$ shows the normalized capture rate $J$ as a function of polymer length $N$ for $\operatorname{SSC}\left(\mathrm{C}_{\text {trans }} / \mathrm{C}_{c i s}=0.9 \mathrm{M} / 0.9 \mathrm{M}=1\right)$ and capture-promoting $\mathrm{ASC}\left(\mathrm{C}_{\text {trans }} / \mathrm{C}_{c i s}\right.$ $=3.6 \mathrm{M} / 0.9 \mathrm{M}=4$ ) conditions. In the SSC condition, as previously observed, ${ }^{28,29,33}$ the capture rate initially sharply increases with DNA length before exhibiting a length-independent behavior. For the experimental conditions used here, the regime-transition polymer length between the barrier-limited and the diffusion-limited regimes is $N^{*} \approx 1 \mathrm{kbp}$. In contrast, the $4 \times$ ASC condition shows a higher normalized capture rate with no such clear dependence on polymer length for the range studied. Only the shortest length tested, at $N=500 \mathrm{bp}$, may indicate the onset of a smooth transition towards a length-dependent regime. Although hard to pinpoint, the capture regime 
transition appears to be shifted toward shorter polymers in capture-promoting ASC conditions as compared to the SSC conditions.

From the capture rate results presented in Figure 2, we conclude that the dynamics of capture in ASC conditions are described by the same physical phenomena as in symmetric case: The barrier-limited and diffusion-limited regimes provide adequate descriptions of the properties of capture under ASC conditions. Moreover, ASC conditions demonstrate modulation of the transition between capture regimes, with capture-opposing gradients $\left(\mathrm{C}_{\text {trans }} / \mathrm{C}_{\text {cis }}<1\right)$ extending the barrier-limited transition to larger polymers and higher voltages, and capture-promoting gradients $\left(\mathrm{C}_{\text {trans }} / \mathrm{C}_{\text {cis }}>1\right)$ reducing the transition to shorter polymers and smaller voltages.

\section{Capture Rate Modulation}

Characterizing the extent to which capture rate is modulated by ASC conditions is of practical value for precise molecular counting applications. The data in Figure 2 and the resulting interpretation that salt gradients alter the capture-regime transitions suggest that the observed capture enhancement between different ASC conditions should also be capture-regime dependent.

To investigate this, we used the $10 \mathrm{kbp}$ dsDNA data from Figure 2e-f to calculate the capture rate enhancement $R_{a s c} / R_{s s c}$ between the capture-promoting ASC $\left(\mathrm{C}_{\text {trans }} / \mathrm{C}_{c i s}=3.6 \mathrm{M} / 0.45\right.$ $\mathrm{M}>1)$ and $\mathrm{SSC}\left(\mathrm{C}_{\text {trans }} / \mathrm{C}_{c i s}=0.45 \mathrm{M} / 0.45 \mathrm{M}=1\right)$ conditions. Figure 3a displays the voltage dependence of the capture rate enhancement. Under a $100 \mathrm{mV}$ bias, the capture rate enhancement is measured to be $\sim 20$-fold. Capture enhancement decreases with increasing voltages until $\approx 400$ $\mathrm{mV}$ where it becomes approximately constant. For $\geq 400 \mathrm{mV}$, the value of the capture enhancement is approximately equivalent to the salt concentration or conductivity ratios of the trans and cis sides. This enhancement value was observed in previous work and is expected for 
the diffusion-limited regime. ${ }^{33,37}$ Its exact value is attributed to the fact that far from the pore, $E_{c i s}$ in ASC conditions is approximately enhanced by a factor of $\sigma_{\text {trans }} / \sigma_{c i s} \approx \mathrm{C}_{\text {trans }} / \mathrm{C}_{\text {cis }}$, when compared to the SSC conditions. Interestingly, the voltage at which $R_{a s c} / R_{s s c}$ plateaus to $\mathrm{C}_{\text {trans }} / \mathrm{C}_{c i s}$ coincides well with $\Delta V^{*}$, the voltage at which capture transitions from barrier- to diffusion-limited under the SSC condition (Figure 2e).

Note that in high salt concentration, electrical conductivity is not strictly linearly related to salt concentration. ${ }^{55}$ Conductivity measurements across the various $\mathrm{LiCl}$ concentrations used here however show that $\sigma_{\text {trans }} / \sigma_{c i s} \approx \mathrm{C}_{\text {trans }} / \mathrm{C}_{c i s}$ is a reasonable approximation (see Section $\mathrm{S} 2$ of the Supporting Information).
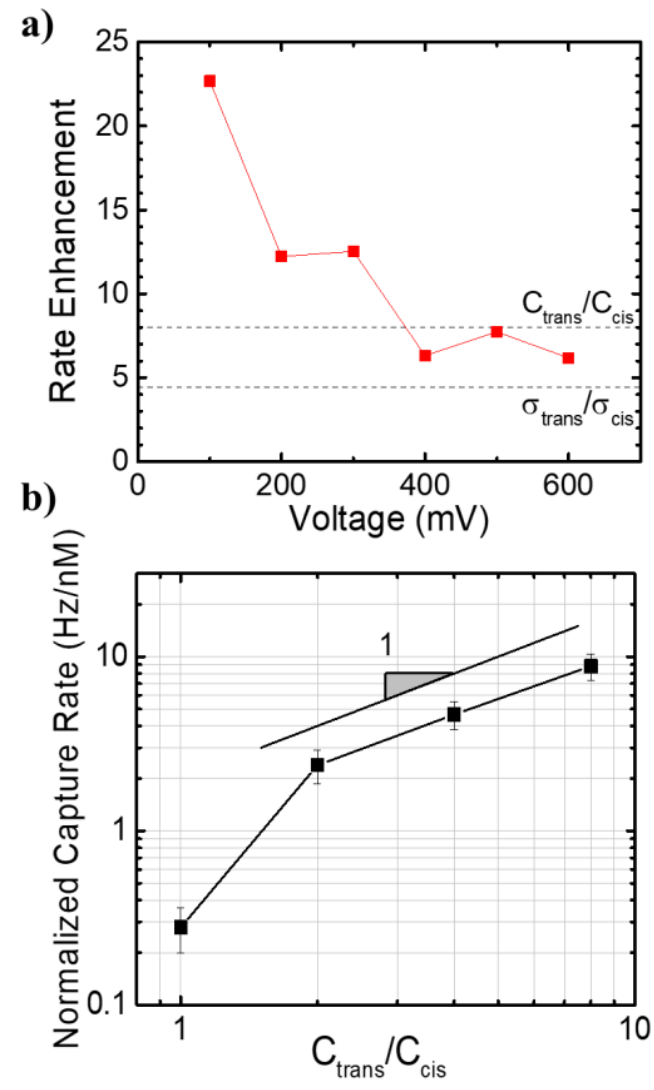

Figure 3. Regime-dependent capture enhancement. (a) Capture rate enhancement $\left(R_{\text {asc }} / R_{s s c}\right)$ versus voltage using $10 \mathrm{kbp}$ capture data in a $5.3 \mathrm{~nm}$ pore from Figure 2. (b) Capture rate versus $\mathrm{C}_{\text {trans }} / \mathrm{C}_{c i s}$ on a $\log -\log$ scale, to 
better show the linear scaling for higher salt concentration ratios. Measurements done in a $5.1 \mathrm{~nm}$ pore, under a 200 $\mathrm{mV}$ bias, using $5 \mathrm{kbp}$ dsDNA with $\mathrm{C}_{c i s}$ fixed at $0.45 \mathrm{M} \mathrm{LiCl}$.

We next measured the capture rate of $5 \mathrm{kbp}$ dsDNA in a $5.1 \mathrm{~nm}$ pore, under a $200 \mathrm{mV}$ bias, with $\mathrm{C}_{\text {cis }}$ fixed at $0.45 \mathrm{M} \mathrm{LiCl}$ and $\mathrm{C}_{\text {trans }}$ ranging from $0.45 \mathrm{M}$ to $3.6 \mathrm{M} \mathrm{LiCl}$. Figure $3 \mathrm{~b}$ shows the normalized capture rate measured for different values of $\mathrm{C}_{\text {trans }} / \mathrm{C}_{\text {cis }}$. As expected from previous publications, ${ }^{33,37}$ capture rate grows monotonically with increasing salt-concentration ratios. Interestingly, a linear dependence of capture rate on $\mathrm{C}_{\text {trans }} / \mathrm{C}_{\text {cis }}$ is observed for $\mathrm{C}_{\text {trans }} / \mathrm{C}_{c i s} \geq 2$, whereas a super-linear trend is observed for smaller salt concentration gradients. As shown in section S3 of the SI, the transition from super-linear to linear dependence on $\mathrm{C}_{\text {trans }} / \mathrm{C}_{\text {cis }}$ was observed under different multiple experimental conditions. ${ }^{37}$

Since both voltage and salt-concentration ratio modulate the cis-side electric field $E_{c i s}$, both Figures $3 \mathrm{a}$ and $3 \mathrm{~b}$ demonstrate that a stronger $E_{c i s}$, which promotes diffusion-limited capture, results in a capture enhancement approximately equal in magnitude to $\mathrm{C}_{\text {trans }} / \mathrm{C}_{c i s}$, whereas a weaker $E_{c i s}$, promoting barrier-limited capture, results in enhancements significantly higher than the salt concentration ratio $\mathrm{C}_{\text {trans }} / \mathrm{C}_{\text {cis }}$. We conclude that, for the data in Figure $3 \mathrm{~b}$ and section $\mathrm{S} 3$ of the SI, polymers in salt gradients $\geq 2$ are in the diffusion-limited capture regime, whereas for smaller salt gradients polymers are in the barrier-limited capture regime.

The concept of regime-dependent capture enhancement appears to be a general feature of transport through nanopores as it also applies to non-linear polymers, such as DNA nanostructures. ${ }^{56}$ In Supporting Information section S4, we show that the scaling of capture rate with $\mathrm{C}_{\text {trans }} / \mathrm{C}_{c i s}$ is different for two similar DNA nanostructures with different intrinsic rigidities. 
Because the nanostructures must deform and bend to pass through the pore, different rigidities result in different free-energy barriers given their different bending energies. The more flexible structure, which experiences a lower free-energy barrier, is seen to scale almost linearly with $\mathrm{C}_{\text {trans }} / \mathrm{C}_{\text {cis }}$, indicating diffusion-limited capture. The more rigid structure, which experiences a higher free-energy barrier, scales super-linearly with the salt concentration ratio, indicating barrierlimited capture.

\section{Translocation Time Scaling}

In the second part of this work, in order to gain more insight into the physics of translocation, we investigate the scaling of translocation time with polymer length and applied voltage in both ASC and SSC conditions. Translocation dynamics are commonly characterized through the scaling coefficients $\alpha$ and $\beta$ of translocation time $\tau$ with polymer length and applied voltage, respectively: $:^{25}$

$$
\tau \sim N^{\alpha} \Delta V^{\beta}
$$

Tension propagation (TP) models dictate that the length-scaling coefficient $\alpha$ should be bounded by 1 and $1+v$, where $v=0.588$ is the Flory exponent for free-draining polymers, and the voltagescaling coefficient $\beta$ should be equal to -1 for free-draining polymers. ${ }^{21,22,51}$

To experimentally verify the scaling of translocation time with polymer length $\tau \sim N^{\alpha}$ under ASC condition $\left(\mathrm{C}_{\text {trans }} / \mathrm{C}_{c i s}=3.6 \mathrm{M} / 0.9 \mathrm{M}=4\right)$, we used dsDNA of lengths ranging from $500 \mathrm{bp}$ to $48 \mathrm{kbp}$ translocating through three $8.5 \mathrm{~nm} \pm 1 \mathrm{~nm}$ diameter pores under a $400 \mathrm{mV}$ bias and compared it to a SSC $\left(\mathrm{C}_{\text {trans }} / \mathrm{C}_{c i s}=0.9 \mathrm{M} / 0.9 \mathrm{M}=1\right)$ control experiment. The measured translocation times are plotted in Figure 4a, which shows that translocation times in capturepromoting ASC conditions are longer than in SSC conditions. Fitting the data to power-scaling 
law functions of the form of Equation 2 yielded scaling coefficients of $\alpha_{s s c}=1.22 \pm 0.02$ for SSC and $\alpha_{\text {asc }}=1.10 \pm 0.07$ for ASC condition (fits shown in Figure S7 in section S5 of the Supporting Information). While the extracted $\alpha_{\text {ssc }}$ coefficient is in very good agreement with a handful of values reported in previous experimental studies, ${ }^{30,57,58}$ the scaling coefficient in ASC conditions, $\alpha_{\text {asc, }}$ is significantly smaller than $\alpha_{\text {ssc }}(P=0.06$, obtained using a Welch t-test). Such a scaling reduction, albeit more drastic, has been reported previously, though when comparing translocation times of DNA entering and exiting a conical nanopore. ${ }^{59}$
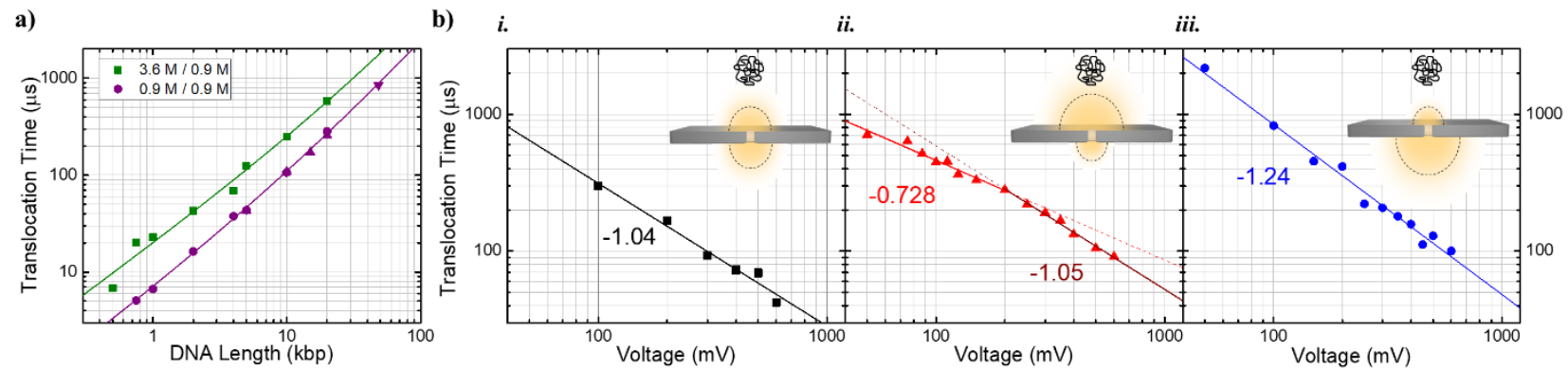

Figure 4. Translocation kinetics in ASC and SSC conditions. (a) Log-log plot of translocation time versus polymer length for symmetric (green squares, $\mathrm{C}_{\text {trans }} / \mathrm{C}_{\text {cis }}=0.9 \mathrm{M} / 0.9 \mathrm{M}$ ) and $4 \mathrm{x}$ capture-promoting ASC (purple circles, $\mathrm{C}_{\text {trans }} / \mathrm{C}_{c i s}=3.6 \mathrm{M} / 0.9 \mathrm{M}$ ) conditions, under $400 \mathrm{mV}$ in $8.5 \pm 1 \mathrm{~nm}$ pore. Each different symbol shape is from a different pore. Continuous curves are fits to Equation 4 (for fits to Equation 2 see SI Figure S7). (b) $i$ ) Translocation time versus voltage in SSC, ii $)$ capture-promoting $\left(\mathrm{C}_{\text {trans }} / \mathrm{C}_{c i s}>1\right)$ and iii $)$ capture-opposing $\left(\mathrm{C}_{\text {trans }} / \mathrm{C}_{c i s}\right.$ $<1)$ ASC conditions. All data is from $10 \mathrm{kbp}$ dsDNA in the same $5.3 \mathrm{~nm}$ pore. Solid lines show the optimal powerlaw fit to the respective data.

Theoretical studies and simulations of tension propagation have shown that a simple power-law expression in the form of Equation 2 is insufficient to capture the length-dependence of translocation times. ${ }^{23,24,50,51,60}$ To incorporate finite polymer-length effects and pore friction, models such as the Iso-Flux Tension Propagation (IFTP) ${ }^{24}$ propose a length-dependence correction of the form 


$$
\tau=A N^{1+v}+B N
$$

where $A$ and $B$ are simple coefficients whose values depend on pore geometry, solution viscosity, and pulling force inside the pore. The first term results from the drag of monomers on the cis-side (a.k.a. cis-monomer friction) and is expected to dominate for long polymers. The second term results from the polymer-pore frictional interactions and is expected to dominate for short polymers. This two-term scaling is equivalent to having a length-dependent scaling coefficient $\alpha(N)$ in Equation 2, where translocations dominated by pore friction $(A \ll B)$ result in $\alpha \approx 1$, and translocations dominated by $c i s$-side monomer $\operatorname{drag}(A \gg B)$ result in $\alpha \approx 1.588$. To experimentally verify the validity of Equation 3, the translocation times of Figure 4a were fitted using the equivalent but more insightful form

$$
\tau=\frac{t_{c}}{2}\left[\left(\frac{N}{N_{c}}\right)^{\kappa}+\frac{N}{N_{c}}\right]
$$

with $t_{c}, N_{c}$ and $\kappa$ as free parameters, allowing the extraction of $\kappa$, the scaling coefficient of cisside monomers, and $N_{c}$ the crossover length at which both the cis-monomer friction term and porefriction term contribute equally to a translocation time of $t_{c}$. Note that $\kappa$ is left as a free parameter in order to verify the IFTP prediction of $\kappa=1.588 .{ }^{24}$ From the optimal fits shown in Figure 4a, we extracted scaling coefficients of $\kappa_{s s c}=1.53 \pm 0.10$ and $\kappa_{a s c}=1.61 \pm 1.49$, and crossover lengths of $N_{C}^{S S C}=10 \pm 6 \mathrm{kbp}$ and $N_{C}^{a s c}=52 \pm 89 \mathrm{kbp}$ in SSC and ASC conditions, respectively. Both $\kappa$ values correspond within error to the expected theoretical value of $\kappa=1+v=1.588$, although with significantly higher uncertainty for $\kappa_{a s c}$. Moreover, the higher value and higher uncertainty of $N_{C}^{a s c}$ indicate that the fitting of ASC data is insensitive to the first term of Equation 4, i.e. cis- 
monomer drag term. A broader range of polymer lengths covering both sides of the transition point would be necessary to reduce the uncertainty on this parameter.

Higher $N_{c}$ value implies that the kinetics of translocation are more dominated by polymerpore interactions in ASC conditions than in SSC conditions. ${ }^{23,24,50}$ This conclusion is consistent with the reduction of the scaling parameter $\alpha$ observed for the simple power-law fits of Equation 2.

We next investigated the scaling of translocation time with applied voltage, $\tau \sim \Delta V^{\beta}$, using the data of Figure 4b, acquired from $10 \mathrm{kbp}$ dsDNA translocating through a $5.3 \mathrm{~nm}$ pore under applied voltages ranging from $50 \mathrm{mV}$ to $600 \mathrm{mV}$ in $\mathrm{SSC}\left(\mathrm{C}_{\text {trans }} / \mathrm{C}_{c i s}=0.45 \mathrm{M} / 0.45 \mathrm{M}=1\right)$, capturepromoting $\mathrm{ASC}\left(\mathrm{C}_{\text {trans }} / \mathrm{C}_{\text {cis }}=3.6 \mathrm{M} / 0.45 \mathrm{M}>1\right)$, and capture-opposing $\mathrm{ASC}\left(\mathrm{C}_{\text {trans }} / \mathrm{C}_{\text {cis }}=0.45\right.$ $\mathrm{M} / 3.2 \mathrm{M}<1$ ) conditions. Figures $4 \mathrm{~b} i$-iii show the translocation times measured at various voltages in the three conditions, where again it can be seen that translocation times in both capturepromoting and capture-opposing ASC conditions are longer than in SSC conditions. Data under the SSC condition (Figure 4b-i) exhibits an inversely proportional voltage-scaling, $\beta=-1.04 \pm$ 0.08 for the entire range of voltages measured, as expected from theory. ${ }^{21,23-25}$ In capturepromoting ASC conditions (Figure 4b-ii), a similar expected scaling of $-1.05 \pm 0.04$ is observed for voltages $\geq 200 \mathrm{mV}$. The scaling value however significantly deviates for lower voltages which instead show a scaling coefficient of $\beta=-0.73 \pm 0.06$. Interestingly, the scaling coefficient from experiments performed in capture-opposing ASC conditions (Figure 4b-iii) also deviates from an inverse scaling with $\beta=-1.24 \pm 0.05$.

Although recent theoretical studies and models predict a scaling of $\beta=-1$ for all voltages, ${ }^{21,23-25}$ the earliest TP report by Saito et $a .^{22}$ introduced three different force-scaling 
regimes for polymer translocation: The strong stretching (under high pulling forces) with scaling $\beta=-1$; The stem-flower regime (under intermediate pulling forces) with scaling $\beta=-2 v /(1+v)$ $\approx-0.74$; The trumpet regime (under weaker pulling forces) with scaling $\beta=-2 /(1+v) \approx-1.26$. The predicted values for the stem-flower and trumpet regimes correspond very well to the experimental values observed here for the capture-opposing and capture-promoting ASC conditions. This excellent agreement is surprising, since this early report is based on a selfidentified erroneous assumption regarding the location of the relevant steady-state velocity (pore

mouth vs. tension front) ${ }^{61}$ Moreover, the derivation of the three regime-dependent scalings did not take into account finite polymer-length effects and pore-polymer friction, ${ }^{50,51}$ which were significant in our experiments as demonstrated by our measurement of $\alpha=1.22$ instead of the expected value of 1.588. The high data density and near perfect agreement make it difficult to point to a simple coincidence, yet we are currently unable to provide a satisfactory explanation as to why our experimental data matches so well with a theoretical model expected to be incorrect. We hope that our findings will motivate further theoretical work on the topic.

\section{Modulation of Translocation Time Statistics}

We now attempt to gain insights into how ASC conditions alter polymer conformations during steps $i i$ and $i i i$ of capture in Figure 1b, the steps prior to translocation during which polymers are elongated and compressed by the external field, respectively. We begin by reporting the dependence of translocation time on salt concentration ratio, which highlights the behavior of the effective pulling force during translocation in various ASC conditions. This is done since the magnitude of the pulling force is expected to highly impact the duration of step iii of capture, where polymers are expected to compress before beginning translocation. 
We measured the translocation times of $10 \mathrm{kbp}$ dsDNA in a $6.0 \mathrm{~nm}$ pore, under a $300 \mathrm{mV}$ bias, with $\mathrm{C}_{\text {cis }}$ fixed at $1.8 \mathrm{M} \mathrm{LiCl}$ and $\mathrm{C}_{\text {trans }}$ ranging from $0.9 \mathrm{M}$ to $3.6 \mathrm{M} \mathrm{LiCl}$. Figure 5a plots the dependence of the mean translocation time $\tau$ on the salt concentration ratio $\mathrm{C}_{\text {trans }} / \mathrm{C}_{c i s}$. Interestingly, for the entire range tested, $\tau$ monotonically increases with $\mathrm{C}_{\text {trans }} / \mathrm{C}_{\text {cis }}$ for both capture-promoting $\left(\mathrm{C}_{\text {trans }} / \mathrm{C}_{c i s}>1\right)$ and capture-opposing $\left(\mathrm{C}_{\text {trans }} / \mathrm{C}_{c i s}<1\right)$ ASC conditions, with $\tau$ seeing a twofold increase in going from $\mathrm{C}_{\text {trans }} / \mathrm{C}_{c i s}=0.5$ to 2 . Translocation time and pulling force being inversely correlated, it can alternatively be concluded that the effective translocation pulling force reduces with $\mathrm{C}_{\text {trans }} / \mathrm{C}_{c i s}$.

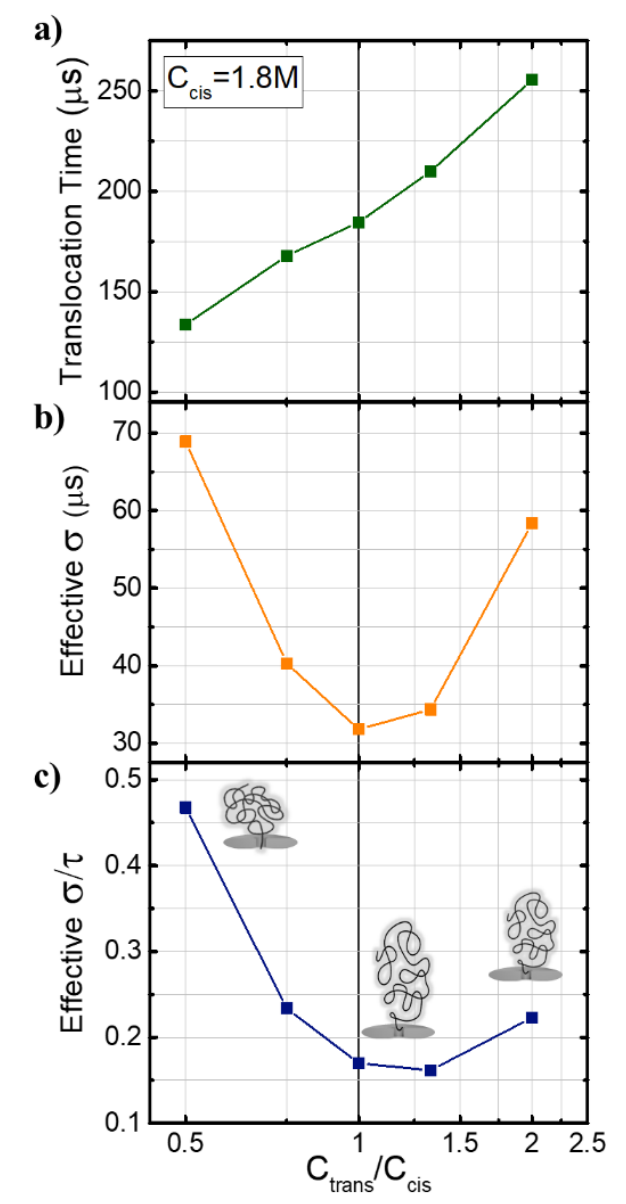

Figure 5. Dependence of $10 \mathrm{kbp}$ translocation time statistics on salt concentration ratios for fixed $\mathrm{C}_{c i s}=1.8 \mathrm{M} \mathrm{LiCl}$ and varying $\mathrm{C}_{\text {trans }}$ under $300 \mathrm{mV}$ in a $6.0 \mathrm{~nm}$ pore. (a) Mean Translocation time, (b) Translocation time standard 
deviation, and (c) Translocation time coefficient of variation versus $\mathrm{C}_{\text {trans }} / \mathrm{C}_{c i s}$ on semi-log $x$-scale. The inset schematics show the deduced polymer elongations in different ASC conditions.

Since the spread of translocation times is a measure of the conformational entropy of polymers at the onset of translocation, ${ }^{57,62}$ we next investigated its dependence on $\mathrm{C}_{\text {trans }} / \mathrm{C}_{c i s}$. To achieve this, we extracted the standard deviation $\sigma$ from the $10 \mathrm{kbp}$ dsDNA translocation time distributions and calculated the coefficient of variation $\sigma / \tau$ in different ASC conditions, as shown in Figures $5 \mathrm{~b}$ and $5 \mathrm{c}$. Unlike translocation times, $\sigma$ exhibits a convex non-monotonic dependence on $\mathrm{C}_{\text {trans }} / \mathrm{C}_{c i s}$, with a minimum located near $\mathrm{C}_{\text {trans }} / \mathrm{C}_{c i s}=1$. As a result, the $\sigma / \tau$ values are asymmetric around $\mathrm{C}_{\text {trans }} / \mathrm{C}_{c i s}=1$ with the capture-promoting ASC conditions resulting in significantly smaller $\sigma / \tau$ values than their inverse capture-opposing conditions.

Previous studies have shown that polymers stretched prior to translocation display reduced conformational entropy (and $\sigma / \tau$ values) due to their more elongated conformations. ${ }^{57,63,64}$ Interpreting $\sigma / \tau$ values as being inversely correlated with the average conformation elongation, Figure $5 \mathrm{c}$ should therefore show a non-monotonic relationship between elongation and salt concentration ratio $\mathrm{C}_{\text {trans }} / \mathrm{C}_{c i s}$ : For a fixed $\mathrm{C}_{c i s}$ and varying $\mathrm{C}_{\text {trans }}$, capture-opposing ASC conditions $\left(\mathrm{C}_{\text {trans }} / \mathrm{C}_{c i s}<1\right)$ result in less elongated polymer conformations than SSC conditions $\left(\mathrm{C}_{\text {trans }} / \mathrm{C}_{c i s}=\right.$ 1), which in turn result in more elongated conformations than capture-promoting ASC conditions $\left(\mathrm{C}_{\text {trans }} / \mathrm{C}_{c i s}>1\right)$, with the maximal elongation occurring near $\mathrm{C}_{\text {trans }} / \mathrm{C}_{c i s}=1$ (see Figure $5 \mathrm{~d}$ ). To further validate the increase of $\sigma / \tau$ in capture-promoting ASC conditions and support the proposed elongation interpretation, the dependence of $\sigma$ and $\sigma / \tau$ on polymer length is shown in Figure S9. 


\section{Insights from Observations}

Multiple claims regarding the nanopore capture and translocation process in ASC and SSC conditions were brought forward in the four previous sections (including that capture-promoting ASC conditions promote diffusion-limited capture due to increased $E_{c i s}$, whereas the opposite is true for capture-opposing ASC conditions, and that translocations under capture-promoting ASC conditions are more dominated by pore friction and are more elongated than under SSC conditions). We now discuss how these observations converge to paint a consistent and complete picture of the nanopore transport process.

First, we address the process by which ASC conditions result in less elongated conformations than in standard SSC conditions. The voltage scalings (Figures 2e-g) and modulations with $\mathrm{C}_{\text {trans }} / \mathrm{C}_{c i s}$ (Figure $3 \mathrm{~b}$ ) of capture rates showed that the external field $E_{c i s}$ increases monotonically with $\mathrm{C}_{\text {trans }} / \mathrm{C}_{\text {cis }}$. Since the external field $E_{c i s}$ magnitude, or rather its gradient $\nabla E_{\text {cis }}$ (see section $\mathrm{S} 1$ of the Supporting Information), is responsible for polymer elongation during the capture step $i$, we thus expect the elongation of polymers throughout step $i i$ to increase monotonically with $\mathrm{C}_{\text {trans }} / \mathrm{C}_{\text {cis }}$.

The increase of translocation times with $\mathrm{C}_{\text {trans }} / \mathrm{C}_{c i s}$ shown in Figure 5a showed that the effective pulling force during translocation $f_{\text {pore }}$ decreases monotonically with $\mathrm{C}_{\text {trans }} / \mathrm{C}_{\text {cis }}$. Note that $f_{\text {pore }}$ is closely related to the duration of the capture step iii: A stronger $f_{\text {pore }}$ should shorten the time required for a polymer segment to enter the pore after its arrival to the pore, and therefore shorten step iii. Given that polymers are compressed against the membrane by $E_{c i s}$ during step $i i$, and that $f_{\text {pore }}$ is inversely correlated with the duration of step iii and $\mathrm{C}_{\text {trans }} / \mathrm{C}_{c i s}$, we expect polymer compression to be more important for higher $\mathrm{C}_{\text {trans }} / \mathrm{C}_{\text {cis }}$. Therefore, we conclude that increasing 
$\mathrm{C}_{\text {trans }} / \mathrm{C}_{c i s}$ has two confounding effects on polymer conformations: it promotes elongation during step $i$, and promotes compression during step iii.

We believe $E_{c i s}$-induced elongation and compression explains the non-monotonic translocation time $\sigma / \tau$ values shown in Figures 5 b,c: For $\mathrm{C}_{\text {trans }} / \mathrm{C}_{c i s} \gg 1$, although polymers arrive at the pore with highly elongated conformations due to high $\nabla E_{c i s}$, they are significantly compressed prior to threading due to the high $E_{c i s}$ and low $f_{\text {pore }}$. For $\mathrm{C}_{\text {trans }} / \mathrm{C}_{c i s}<1$, polymers are not significantly elongated by $\nabla E_{c i s}$ when arriving at the pore, nor are they significantly compressed due to the weaker $E_{c i s}$ and stronger $f_{\text {pore }}$. Note that there exists a value of $\mathrm{C}_{\text {trans }} / \mathrm{C}_{c i s}$ that naturally results in maximal elongation during translocation, by optimally balancing $\nabla E_{c i s^{-}}$ induced pre-stretching and $E_{c i s}$-induced compression. For the current experimental conditions, this value empirically occurs at a salt ratio slightly higher than 1 , as demonstrated by the minimal $\sigma / \tau$ value of Figure 5c. This minimum in translocation time spread, surprisingly close to $\mathrm{C}_{\text {trans }} / \mathrm{C}_{c i s}=$ 1, corresponds to the optimized experimental condition under which molecules can be characterized and separated.

To further support polymers being less elongated in ASC conditions than in SSC, we note that more elongated conformations are expected to result in more significant cis-side monomer friction throughout the translocation steps $i v$ and $v .^{24,62}$ This is simply due to more elongated conformations having more monomers under tension, and therefore in motion, soon after translocation begins, with the limiting case being a completely stretched-out polymer with end monomers moving almost instantly as translocation begins. The concept of ASC conditions inducing less-elongated conformations is notably consistent with the observations drawn from Figure 4a, where in fitting $\tau(N)$ to Equations 2 and 4, we concluded that translocations in ASC conditions $\left(\mathrm{C}_{\text {trans }} / \mathrm{C}_{c i s}=3.6 \mathrm{M} / 0.9 \mathrm{M}\right)$ were more pore-friction dominated than in $\mathrm{SSC}$, or 
equivalently that cis-side monomer friction was less dominant than in SSC. We therefore believe that the scaling coefficient reduction observed in ASC conditions is a direct consequence of polymers being less elongated prior to translocation.

The experimental results shown in this work allow us to comment on the mechanisms that are believed to be important for slowing down translocation in ASC conditions, namely the reduction of $\Delta V_{\text {pore }},{ }^{33}$ and the motion-opposing electro-osmotic flow due to cation buildup inside the pore. ${ }^{36}$ In section S6 of the Supporting Information, we further discuss how the local salt concentration inside the pore is expected to control the translocation time modulation in ASC conditions, just as bulk salt concentration affects translocation times. ${ }^{42}$

Lastly, while the close agreement between experimentally measured (Figure 4b) and theoretically predicted scalings ${ }^{22}$ of translocation time with applied voltage $\Delta V$ is surprising, ${ }^{61}$ the occurrence of non-inverse $(\beta=-1)$ scalings observed in ASC conditions can be understood by considering how the effective pulling force $f_{\text {pore }}$ is altered both by the applied voltage, and by ASC conditions. First, $f_{\text {pore }}$ increases with $\Delta V$, as revealed by translocation times reducing with $\Delta V$ (Figure $4 \mathrm{~b}$ ). Moreover, SSC conditions result in higher $f_{\text {pore }}$ compared to both capturepromoting and capture-opposing ASC conditions, as illustrated by the translocation times always becoming shorter with increasing voltages in Figure $4 \mathrm{~b}$ (and in Figure S8b of the Supporting Information). The strong stretching regime ( $\beta=-1$, high pulling force) measured in SSC conditions, and the transition from stem-flower regime $(\beta=-2 v /(1+v)$, intermediate pulling force) at low voltages to strong stretching regime at higher voltages in capture-promoting ASC conditions are therefore in line with theoretical expectations. Although the trumpet regime $(\beta=-$ $2 /(1+v)$, weak pulling force) in capture-opposing ASC conditions is consistent with weaker $f_{\text {pore }}$, 
the discrepancy between capture-promoting and -opposing ASC conditions may highlight the effects of initial polymer conformations, wherein capture-promoting ASC conditions are expected to result in more elongated conformations than in capture-opposing conditions (see Figure 5c).

\section{Conclusion}

We presented an extensive description of DNA capture and translocation dynamics through characterization of the dependence of capture rate, translocation time statistics, and folding kinetics (shown in section S8 of Supporting Information), on voltage, polymer length, and salt concentration gradient.

We showed that the underlying physics describing the transport process in asymmetric salt concentration (ASC) conditions is the same as in symmetric salt concentrations (SSC). The barrierand diffusion-limited capture regimes combined with tension propagation principles fully describe the capture and translocation process. The promotions of diffusion- or barrier-limited regimes in capture-promoting or -opposing ASC conditions respectively demonstrated that the cis-side electric field plays a crucial role in determining capture kinetics. The higher order statistics of translocation time distributions further confirmed experimentally that $\nabla E_{c i s}$ and $E_{c i s}$ are responsible for elongating and compressing polymers before and after reaching the pore mouth, respectively.

This work confirmed the previously published conclusions ${ }^{33,35-41}$ that detecting molecules from the low salt-concentration side of a salt gradient increases capture rate, slows down translocation, and increases the signal-to-noise ratio through deeper blockage depths. Although these are ideal features for nanopore sensing, we also observed an increase in folded translocations (section S8 of the Supporting Information), an increase in translocation time spread (Figure 5a), 
and significantly more frequent pore clogs. The increased folding and translocation time spread can be undesirable for applications requiring single-file passage, such as DNA-carrier-based bioassays and data storage applications which require mapping with the highest precision, objects bound to linear polymers. The biggest drawback however to ASC conditions may probably be the likelihood of permanent pore clogs, occurring more frequently than in symmetric conditions, and therefore significantly reducing the lifespan and usability of nanopores. For asymmetric salt concentration conditions to be of practical use in different applications, this issue should be

addressed, possibly employing different coating strategies and types of surface chemistries ${ }^{65,66}$ to control polymer pore interactions.

Finally, we hope that our results will provide motivation for further theoretical work on the capture and translocation processes. We believe that consideration of the external electric field and its role in elongating and compressing polymers should help bridge theoretical and experimental efforts. Namely, elucidating the impact of field-induced polymer conformations on the translocation time scaling with polymer length $\tau(N)$ and applied voltage $\tau(\Delta V)$ will help develop a thorough understanding of the nanopore transport process, which will be essential in guiding development of nanopore-based applications in numerous critical fields.

\section{Experimental Methods}

Nanopore Fabrication. Nanopores were fabricated in $10 \mathrm{~nm}$ thick SiN membranes purchased from Norcada Inc. (NBPX5004Z-60O-Hi RES) using controlled breakdown, following the protocols and procedures outlined in detail in Waugh et al. ${ }^{67}$ Concisely, pores were fabricated in 
$1 \mathrm{M} \mathrm{KCl} \mathrm{pH} 8$ using a linear voltage ramp, followed by conditioning to enlarge using 3 second 3 V pulses in $3.6 \mathrm{M} \mathrm{LiCl} \mathrm{pH} 8$, until the desired pore size was reached. ${ }^{68}$

DNA Translocation Experiments. Ionic current traces were sampled using a Chimera VC100 at 4.167 MHz. After establishing a difference in salt concentration across the nanopore, a minimum of 30 minutes was allowed to elapse before applying a voltage and recording translocation events, to ensure a time-independent I-V curve (see Section S9 of Supporting Information), which we attribute to salts mixing inside the nanopore and reaching a steady state. The current signal is recorded for 10-15 minutes depending on the observed capture rate with the aim of collecting at least 300 events. During the experiment, a zapping function was enabled which inverted the bias polarity for 3 seconds if the open pore conductance decreased by $>5 \%$ its original value indicating undesired clogging of the pore. Data acquired during zapping was ignored for analysis. DNA concentrations were ascertained using a spectrophotometer (Biotek Epoch 2). For experiments on a single pore, for which multiple voltages or DNA lengths were sampled, the experiment order was randomized as to not have time-dependent pore characteristics (e.g. growth) be responsible for changes in capture and translocation kinetics, instead of the varying voltage or DNA length.

Data Analysis. Current trace data is analyzed using a custom implementation of the CUSUM+ and adept2state algorithms (see https://github.com/shadowk29/CUSUM) ${ }^{69}$ Since capture inherently obeys Poisson process statistics, capture rates are extracted by fitting the histogram of the logarithm of inter-event times to the log-transform of the Poisson distribution function. A detailed description of the accuracy of this capture rate fitting method and other similar methods in different experimental conditions is provided in our previous work. ${ }^{29}$ The mean translocation time of unfolded DNA and its standard deviation are extracted from experimental data in one of two ways: 1) Since unfolded translocation times are sufficiently well described by a log-normal 
distribution, a Gaussian fit is applied to the histogram of the logarithm of the translocation times, provided enough unfolded translocations are observed. The mean and standard deviation of translocation times are then calculated using well-known lognormal properties; 2) Since asymmetric salts significantly promotes folded translocations (see Figure S10), large sample sizes of unfolded events can be difficult to obtain. In such cases, we define the effective translocation time $\tau_{\text {eff }}$ to be the ratio of the equivalent charge deficit (ECD) and the single dsDNA blockage level. Here, ECD denotes the integral of the ionic blockage with respect to the open-pore baseline. This approach mathematically unravels the blockage trace and can be used to faithfully increase the sample size of the mean unfolded translocation time. Section S10 of the Supporting Information validates this second method by demonstrating that both methods quantitatively agree on the standard deviation of the translocation time distribution of a highly populated data set.

\section{ASSOCIATED CONTENT}

\section{Supporting Information}

The following files are available free of charge:

Additional information regarding the three-resistor model and its resulting conductance, potential and electric fields in ASC conditions, the non-linearity of salt-concentration and conductivity, the regime-dependent capture enhancement viewed through the dependence of capture rate on salt gradients if linear DNA and DNA nanostructures, the different fits of translocation time and DNA length scaling, the mechanisms responsible for translocation time modulation, the DNA-length dependence of higher order translocation time statistics, the folding statistics, IV stabilization when going from SSC to ASC conditions, and regarding the methods 
for extracting the standard deviation of translocation times (PDF)

\section{AUTHOR INFORMATION}

\section{Corresponding Author}

*E-mail: tcossa@uOttawa.ca

\section{Author Contributions}

\$M.C. and L.P. contributed equally.

\section{Funding Sources}

The authors would like to acknowledge the support of the Natural Sciences and Engineering Research Council of Canada (NSERC), through funding from grant \#CRDPJ 530554-18.

\section{ACKNOWLEDGMENT}

The authors would like to thank Kyle Briggs and Gary Slater for fruitful discussions.

\section{ABBREVIATIONS}

ASC, asymmetric salt concentrations; SSC, symmetric salt concentrations; TP, tension propagation; IFTP, Iso-flux tension propagation.

\section{REFERENCES}

(1) Deamer, D.; Akeson, M.; Branton, D. Three Decades of Nanopore Sequencing. Nat. Biotechnol. 2016, 34 (5), 518-524. https://doi.org/10.1038/nbt.3423.

(2) Logsdon, G. A.; Vollger, M. R.; Eichler, E. E. Long-Read Human Genome Sequencing and Its Applications. Nat. Rev. Genet. 2020, 21 (10), 597-614. https://doi.org/10.1038/s41576020-0236-x.

(3) Garalde, D. R.; Snell, E. A.; Jachimowicz, D.; Sipos, B.; Lloyd, J. H.; Bruce, M.; Pantic, 
N.; Admassu, T.; James, P.; Warland, A.; Jordan, M.; Ciccone, J.; Serra, S.; Keenan, J.; Martin, S.; McNeill, L.; Wallace, E. J.; Jayasinghe, L.; Wright, C.; Blasco, J.; Young, S.; Brocklebank, D.; Juul, S.; Clarke, J.; Heron, A. J.; Turner, D. J. Highly Parallel Direct RNA Sequencing on an Array of Nanopores. Nat. Methods 2018, 15 (3), 201-206. https://doi.org/10.1038/nmeth.4577.

(4) Branton, D.; Deamer, D. W.; Marziali, A.; Bayley, H.; Benner, S. A.; Butler, T.; Di Ventra, M.; Garaj, S.; Hibbs, A.; Huang, X.; Jovanovich, S. B.; Krstic, P. S.; Lindsay, S.; Ling, X. S.; Mastrangelo, C. H.; Meller, A.; Oliver, J. S.; Pershin, Y. V.; Ramsey, J. M.; Riehn, R.; Soni, G. V.; Tabard-Cossa, V.; Wanunu, M.; Wiggin, M.; Schloss, J. A. The Potential and Challenges of Nanopore Sequencing. Nat. Biotechnol. 2008, 26 (10), 1146-1153. https://doi.org/10.1038/nbt.1495.

(5) Lindsay, S. The Promises and Challenges of Solid-State Sequencing. Nat. Nanotechnol. 2016, 11 (2), 109-111. https://doi.org/10.1038/nnano.2016.9.

(6) Xue, L.; Yamazaki, H.; Ren, R.; Wanunu, M.; Ivanov, A. P.; Edel, J. B. Solid-State Nanopore Sensors. Nat. Rev. Mater. 2020, 5 (12), 931-951. https://doi.org/10.1038/s41578020-0229-6.

(7) Bell, N. A. W.; Keyser, U. F. Digitally Encoded DNA Nanostructures for Multiplexed, Single-Molecule Protein Sensing with Nanopores. Nat. Nanotechnol. 2016, 11 (7), 645651. https://doi.org/10.1038/nnano.2016.50.

(8) Chuah, K.; Wu, Y.; Vivekchand, S. R. C.; Gaus, K.; Reece, P. J.; Micolich, A. P.; Gooding, J. J. Nanopore Blockade Sensors for Ultrasensitive Detection of Proteins in Complex Biological Samples. Nat. Commun. 2019, 10 (1). https://doi.org/10.1038/s41467-01910147-7.

(9) Sze, J. Y. Y.; Ivanov, A. P.; Cass, A. E. G.; Edel, J. B. Single Molecule Multiplexed Nanopore Protein Screening in Human Serum Using Aptamer Modified DNA Carriers. Nat. Commun. 2017, 8 (1), 1-10. https://doi.org/10.1038/s41467-017-01584-3.

(10) Raveendran, M.; Lee, A. J.; Sharma, R.; Wälti, C.; Actis, P. Rational Design of DNA Nanostructures for Single Molecule Biosensing. Nat. Commun. 2020, 11 (1), 1-9. https://doi.org/10.1038/s41467-020-18132-1.

(11) Morin, T. J.; McKenna, W. L.; Shropshire, T. D.; Wride, D. A.; Deschamps, J. D.; Liu, X.; Stamm, R.; Wang, H.; Dunbar, W. B. A Handheld Platform for Target Protein Detection and Quantification Using Disposable Nanopore Strips. Sci. Rep. 2018, 8 (1), 14834. https://doi.org/10.1038/s41598-018-33086-7.

(12) Varongchayakul, N.; Song, J.; Meller, A.; Grinstaff, M. W. Single-Molecule Protein Sensing in a Nanopore: A Tutorial. Chem. Soc. Rev. 2018, 47 (23), 8512-8524. https://doi.org/10.1039/c8cs00106e.

(13) Yusko, E. C.; Bruhn, B. R.; Eggenberger, O. M.; Houghtaling, J.; Rollings, R. C.; Walsh, N. C.; Nandivada, S.; Pindrus, M.; Hall, A. R.; Sept, D.; Li, J.; Kalonia, D. S.; Mayer, M. Real-Time Shape Approximation and Fingerprinting of Single Proteins Using a Nanopore. 
Nat. Nanotechnol. 2016, 12 (4), 360-367. https://doi.org/10.1038/nnano.2016.267.

(14) Ouldali, H.; Sarthak, K.; Ensslen, T.; Piguet, F.; Manivet, P.; Pelta, J.; Behrends, J. C.; Aksimentiev, A.; Oukhaled, A. Electrical Recognition of the Twenty Proteinogenic Amino Acids Using an Aerolysin Nanopore. Nat. Biotechnol. 2020, 38 (2), 176-181. https://doi.org/10.1038/s41587-019-0345-2.

(15) Nivala, J.; Marks, D. B.; Akeson, M. Unfoldase-Mediated Protein Translocation through an $\alpha$-Hemolysin Nanopore. Nat. Biotechnol. 2013, $31 \quad$ (3), 247-250. https://doi.org/10.1038/nbt.2503.

(16) Alfaro, J. A.; Bohländer, P.; Dai, M.; Filius, M.; Howard, C. J.; van Kooten, X. F.; Ohayon, S.; Pomorski, A.; Schmid, S.; Aksimentiev, A.; Anslyn, E. V.; Bedran, G.; Cao, C.; Chinappi, M.; Coyaud, E.; Dekker, C.; Dittmar, G.; Drachman, N.; Eelkema, R.; Goodlett, D.; Hentz, S.; Kalathiya, U.; Kelleher, N. L.; Kelly, R. T.; Kelman, Z.; Kim, S. H.; Kuster, B.; Rodriguez-Larrea, D.; Lindsay, S.; Maglia, G.; Marcotte, E. M.; Marino, J. P.; Masselon, C.; Mayer, M.; Samaras, P.; Sarthak, K.; Sepiashvili, L.; Stein, D.; Wanunu, M.; Wilhelm, M.; Yin, P.; Meller, A.; Joo, C. The Emerging Landscape of Single-Molecule Protein Sequencing Technologies. Nat. Methods 2021, 18 (6), 604-617. https://doi.org/10.1038/s41592-021-01143-1.

(17) Chen, K.; Kong, J.; Zhu, J.; Ermann, N.; Predki, P.; Keyser, U. F. Digital Data Storage Using DNA Nanostructures and Solid-State Nanopores. Nano Lett. 2019. https://doi.org/10.1021/acs.nanolett.8b04715.

(18) Cao, C.; Krapp, L. F.; Ouahabi, A. Al; König, N. F.; Cirauqui, N.; Radenovic, A.; Lutz, J. F.; Peraro, M. D. Aerolysin Nanopores Decode Digital Information Stored in Tailored Macromolecular Analytes. Sci. $A d v . \quad \mathbf{2 0 2 0}, \quad 6 \quad(50), \quad 2-10$. https://doi.org/10.1126/sciadv.abc2661.

(19) Muthukumar, M. Theory of Capture Rate in Polymer Translocation. J. Chem. Phys. 2010, 132 (19). https://doi.org/10.1063/1.3429882.

(20) Rowghanian, P.; Grosberg, A. Y. Electrophoretic Capture of a DNA Chain into a Nanopore. Phys. Rev. E - Stat. Nonlinear, Soft Matter Phys. 2013, 87 (4), 1-8. https://doi.org/10.1103/PhysRevE.87.042722.

(21) Rowghanian, P.; Grosberg, A. Y. Force-Driven Polymer Translocation through a Nanopore: An Old Problem Revisited. J. Phys. Chem. B 2011, 115 (48), 14127-14135. https://doi.org/10.1021/jp204014r.

(22) Saito, T.; Sakaue, T. Dynamical Diagram and Scaling in Polymer Driven Translocation. Eur. Phys. J. E 2011, 34 (12), 135. https://doi.org/10.1140/epje/i2011-11135-3.

(23) Ikonen, T.; Bhattacharya, A.; Ala-Nissila, T.; Sung, W. Influence of Non-Universal Effects on Dynamical Scaling in Driven Polymer Translocation. J. Chem. Phys 2012, 137, 85101. https://doi.org/10.1063/1.4742188.

(24) Sarabadani, J.; Ikonen, T.; Ala-Nissila, T. Iso-Flux Tension Propagation Theory of Driven 
Polymer Translocation: The Role of Initial Configurations. J. Chem. Phys. 2014, 141 (21), 214907. https://doi.org/10.1063/1.4903176.

(25) Palyulin, V. V.; Ala-Nissila, T.; Metzler, R. Polymer Translocation: The First Two Decades and the Recent Diversification. Soft Matter 2014, 10 (45), 9016-9037. https://doi.org/10.1039/c4sm01819b.

(26) Dubbeldam, J. L. A.; Rostiashvili, V. G.; Milchev, A.; Vilgis, T. A. Forced Translocation of a Polymer: Dynamical Scaling versus Molecular Dynamics Simulation. Phys. Rev. E 2012, 85 (4), 41801. https://doi.org/10.1103/PhysRevE.85.041801.

(27) Davenport, M.; Healy, K.; Pevarnik, M.; Teslich, N.; Cabrini, S.; Morrison, A. P.; Siwy, Z. S.; Létant, S. E. The Role of Pore Geometry in Single Nanoparticle Detection. ACS Nano 2012, 6 (9), 8366-8380. https://doi.org/10.1021/nn303126n.

(28) Bell, N. A. W.; Muthukumar, M.; Keyser, U. F. Translocation Frequency of DoubleStranded DNA through a Solid-State Nanopore. Phys. Rev. E 2016, 93 (2). https://doi.org/10.1103/PhysRevE.93.022401.

(29) Charron, M.; Briggs, K.; King, S.; Waugh, M.; Tabard-Cossa, V. Precise DNA Concentration Measurements with Nanopores by Controlled Counting. Anal. Chem. 2019, 91 (19), acs.analchem.9b01900. https://doi.org/10.1021/acs.analchem.9b01900.

(30) Storm, A. J.; Storm, C.; Chen, J.; Zandbergen, H.; Joanny, J. F.; Dekker, C. Fast DNA Translocation through a Solid-State Nanopore. Nano Lett. 2005, 5 (7), 1193-1197. https://doi.org/10.1021/n1048030d.

(31) van Dorp, S.; Keyser, U. F.; Dekker, N. H.; Dekker, C.; Lemay, S. G. Origin of the Electrophoretic Force on DNA in Solid-State Nanopores. Nat. Phys. 2009, 5 (5), 347-351. https://doi.org/10.1038/nphys1230.

(32) Carson, S.; Wilson, J.; Aksimentiev, A.; Wanunu, M. Smooth DNA Transport through a Narrowed Pore Geometry. Biophys. J. 2014, 107 (10), 2381-2393. https://doi.org/10.1016/j.bpj.2014.10.017.

(33) Wanunu, M.; Morrison, W.; Rabin, Y.; Grosberg, A. Y.; Meller, A. Electrostatic Focusing of Unlabelled DNA into Nanoscale Pores Using a Salt Gradient. Nat. Nanotechnol. 2010, 5 (2), 160-165. https://doi.org/10.1038/nnano.2009.379.

(34) Chen, K.; Jou, I.; Ermann, N.; Muthukumar, M.; Keyser, U. F.; Bell, N. A. W. Dynamics of Driven Polymer Transport through a Nanopore. Nat. Phys. 2021. https://doi.org/10.1038/s41567-021-01268-2.

(35) Chou, T. Enhancement of Charged Macromolecule Capture by Nanopores in a Salt Gradient. J. Chem. Phys. 2009, 131 (3). https://doi.org/10.1063/1.3170952.

(36) He, Y.; Tsutsui, M.; Scheicher, R. H.; Fan, C.; Taniguchi, M.; Kawai, T. Mechanism of How Salt-Gradient-Induced Charges Affect the Translocation of DNA Molecules through a Nanopore. Biophys. J. 2013, 105 (3), 776-782. https://doi.org/10.1016/j.bpj.2013.05.065. 
(37) Jeon, B. J.; Muthukumar, M. Polymer Capture by $\alpha$-Hemolysin Pore upon Salt Concentration Gradient. J. Chem. Phys. 2014, 140 (1). https://doi.org/10.1063/1.4855075.

(38) He, Y.; Tsutsui, M.; Scheicher, R. H.; Miao, X. S.; Taniguchi, M. Salt-Gradient Approach for Regulating Capture-to-Translocation Dynamics of DNA with Nanochannel Sensors. ACS Sensors 2016, 1 (6), 807-816. https://doi.org/10.1021/acssensors.6b00176.

(39) Ivica, J.; Williamson, P. T. F.; De Planque, M. R. R. Salt Gradient Modulation of MicroRNA Translocation through a Biological Nanopore. 2017. https://doi.org/10.1021/acs.analchem.7b01246.

(40) Nova, I. C.; Derrington, I. M.; Craig, J. M.; Noakes, M. T.; Tickman, I.; Doering, K.; Higinbotham, H.; Laszlo, A. H.; Gundlach, J. H. Investigating Asymmetric Salt Profiles for Nanopore DNA Sequencing with Biological Porin MspA. 2017, No. Fig 1, 1-14.

(41) Bello, J.; Mowla, M.; Troise, N.; Soyring, J.; Borgesi, J.; Shim, J. Increased Dwell Time and Occurrence of DsDNA Translocation Events through Solid State Nanopores by LiCl $\begin{array}{lllll}\text { Concentration. } & \text { Electrophoresis } & \text { 2019, } & 40 & \text { (7), }\end{array}$ https://doi.org/10.1002/elps.201800426.

(42) Kowalczyk, S. W.; Wells, D. B.; Aksimentiev, A.; Dekker, C. Slowing down DNA Translocation through a Nanopore in Lithium Chloride. Nano Lett. 2012, 12 (2), 10381044. https://doi.org/10.1021/n1204273h.

(43) Verschueren, D. V.; Jonsson, M. P.; Dekker, C. Temperature Dependence of DNA Translocations through Solid-State Nanopores. Nanotechnology 2015, 26 (23), 234004. https://doi.org/10.1088/0957-4484/26/23/234004.

(44) Freedman, K. J.; Raza Haq, S.; Fletcher, M. R.; Foley, J. P.; Jemth, P.; Edel, J. B.; Jun Kim, M. Nonequilibrium Capture Rates Induce Protein Accumulation and Enhanced Adsorption to Solid-State Nanopores. 2020, 17, 53. https://doi.org/10.1021/nn5062645.

(45) Farahpour, F.; Maleknejad, A.; Varnik, F.; Ejtehadi, M. R. Chain Deformation in Translocation Phenomena. Soft Matter 2013, 9 (9), 2750-2759. https://doi.org/10.1039/c2sm27416g.

(46) Vollmer, S. C.; De Haan, H. W. Translocation Is a Nonequilibrium Process at All Stages: Simulating the Capture and Translocation of a Polymer by a Nanopore. J. Chem. Phys. 2016, 145 (15). https://doi.org/10.1063/1.4964630.

(47) Qiao, L.; Slater, G. W. Ratcheting Charged Polymers through Symmetric Nanopores Using Pulsed Fields: Designing a Low Pass Filter for Concentrating DNA. 2021, 5, 1-6.

(48) Nakane, J.; Akeson, M.; Marziali, A. Evaluation of Nanopores as Candidates for Electronic Analyte Detection. Electrophoresis 2002, $23 \quad$ (16), 2592-2601. https://doi.org/10.1002/1522-2683(200208)23:16<2592::AID-ELPS2592>3.0.CO;2-L.

(49) Gershow, M.; Golovchenko, J. A. Recapturing and Trapping Single Molecules with a SolidState Nanopore. Nat. Nanotechnol. 2007, 2 (12), 775-779. https://doi.org/10.1038/nnano.2007.381. 
(50) Ikonen, T.; Bhattacharya, A.; Ala-Nissila, T.; Sung, W. Influence of Pore Friction on the Universal Aspects of Driven Polymer Translocation. EPL (Europhysics Lett. 2013, 103 (3), 38001. https://doi.org/10.1209/0295-5075/103/38001.

(51) Ikonen, T.; Bhattacharya, A.; Ala-Nissila, T.; Sung, W. Unifying Model of Driven Polymer Translocation. Phys. Rev. E - Stat. Nonlinear, Soft Matter Phys. 2012, 85 (5), 1-7. https://doi.org/10.1103/PhysRevE.85.051803.

(52) Sakaue, T. Dynamics of Polymer Translocation: A Short Review with an Introduction of Weakly-Driven Regime. Polymers (Basel). 2016, 8 (12), 1-12. https://doi.org/10.3390/polym8120424.

(53) Meller, A.; Nivon, L.; Branton, D. Voltage-Driven DNA Translocations through a Nanopore. Phys. Rev. Lett. 2001, $86 \quad$ (15), 3435-3438. https://doi.org/10.1103/PhysRevLett.86.3435.

(54) Stellwagen, N. C.; Gelfi, C.; Righetti, P. G. The Free Solution Mobility of DNA. Biopolymers 1997, $42 \quad$ (6), 687-703. https://doi.org/10.1002/(SICI)10970282(199711)42:6<687::AID-BIP7>3.0.CO;2-Q.

(55) Tanaka, K.; Reita Tamamushi. A Physico-Chemical Study of Concentrated Aqueous Solutions of Lithium Chloride. Zeitschrift für Naturforsch. A 1991, 8 (5), 55.

(56) He, L.; Karau, P.; Tabard-Cossa, V. Fast Capture and Multiplexed Detection of Short MultiArm DNA Stars in Solid-State Nanopores. Nanoscale 2019, 11 (35), 16342-16350. https://doi.org/10.1039/c9nr04566j.

(57) Briggs, K.; Madejski, G.; Magill, M.; Kastritis, K.; de Haan, H. W.; McGrath, J. L.; TabardCossa, V. DNA Translocations through Nanopores under Nanoscale Preconfinement. Nano Lett. 2018, 18 (2), 660-668. https://doi.org/10.1021/acs.nanolett.7b03987.

(58) Mihovilovic, M.; Hagerty, N.; Stein, D. Statistics of DNA Capture by a Solid-State Nanopore. Phys. Rev. Lett. 2013, $110 \quad$ (2), 1-5. https://doi.org/10.1103/PhysRevLett.110.028102.

(59) Bell, N. A. W.; Chen, K.; Ghosal, S.; Ricci, M.; Keyser, U. F. Asymmetric Dynamics of DNA Entering and Exiting a Strongly Confining Nanopore. Nat. Commun. 2017, 8 (1), 380. https://doi.org/10.1038/s41467-017-00423-9.

(60) Sarabadani, J.; Ikonen, T.; Mökkönen, H.; Ala-Nissila, T.; Carson, S.; Wanunu, M. Driven Translocation of a Semi-Flexible Polymer through a Nanopore. Sci. Rep. 2017, 7 (1), 1-8. https://doi.org/10.1038/s41598-017-07227-3.

(61) Saito, T.; Sakaue, T. Erratum to: Dynamical Diagram and Scaling in Polymer Driven Translocation. Eur. Phys. J. E 2012, 35 (11), 0-1. https://doi.org/10.1140/epje/i201212125-7.

(62) Lu, B.; Albertorio, F.; Hoogerheide, D. P.; Golovchenko, J. A. Origins and Consequences of Velocity Fluctuations during DNA Passage through a Nanopore. Biophys. J. 2011, 101 (1), 70-79. https://doi.org/10.1016/j.bpj.2011.05.034. 
(63) de Haan, H. W.; Sean, D.; Slater, G. W. Reducing the Variance in the Translocation Times by Prestretching the Polymer. Phys. Rev. E 2018, 98 (2), 22501. https://doi.org/10.1103/PhysRevE.98.022501.

(64) Sean, D.; de Haan, H. W.; Slater, G. W. Translocation of a Polymer through a Nanopore Starting from a Confining Nanotube. Electrophoresis 2015, 36 (5), 682-691. https://doi.org/10.1002/elps.201400418.

(65) Bandara, Y. M. N. D. Y.; Karawdeniya, B. I.; Hagan, J. T.; Chevalier, R. B.; Dwyer, J. R. Chemically Functionalizing Controlled Dielectric Breakdown Silicon Nitride Nanopores by Direct Photohydrosilylation. ACS Appl. Mater. Interfaces 2019, 11 (33), 30411-30420. https://doi.org/10.1021/acsami.9b08004.

(66) Eggenberger, O. M.; Ying, C.; Mayer, M. Surface Coatings for Solid-State Nanopores. Nanoscale 2019, 11 (42), 19636-19657. https://doi.org/10.1039/C9NR05367K.

(67) Waugh, M.; Briggs, K.; Gunn, D.; Gibeault, M.; King, S.; Ingram, Q.; Jimenez, A. M.; Berryman, S.; Lomovtsev, D.; Andrzejewski, L.; Tabard-Cossa, V. Solid-State Nanopore Fabrication by Automated Controlled Breakdown. Nat. Protoc. 2020, 15 (1), 122-143. https://doi.org/10.1038/s41596-019-0255-2.

(68) Beamish, E.; Kwok, H.; Tabard-Cossa, V.; Godin, M. Precise Control of the Size and Noise of Solid-State Nanopores Using High Electric Fields. Nanotechnology 2012, 23 (40), 405301. https://doi.org/10.1088/0957-4484/23/40/405301.

(69) Forstater, J. H. J. H.; Briggs, K.; Robertson, J. W. F. F. J. W. F.; Ettedgui, J.; Marie-Rose, O.; Vaz, C.; Kasianowicz, J. J. J.; Tabard-Cossa, V.; Balijepalli, A. MOSAIC: A Modular Single-Molecule Analysis Interface for Decoding Multistate Nanopore Data. Anal. Chem. 2016, 88 (23), 11900-11907. https://doi.org/10.1021/acs.analchem.6b03725. 